\title{
Generalisation of the Frobenius Formula in the Theory of Block Operators on Normed Spaces
}

\author{
Nikolai A. Sidorov ${ }^{1}$, Aliona I. Dreglea ${ }^{2}$ (D) and Denis N. Sidorov ${ }^{1,2,3, *(\mathbb{D})}$ \\ 1 Institute of Mathematics and Information Technologies, Irkutsk State University, 664025 Irkutsk, Russia; \\ sidorov@math.isu.runnet.ru \\ 2 Industrial Mathematics Laboratory, Baikal School of BRICS, Irkutsk National Research Technical University, \\ 664074 Irkutsk, Russia; adreglea@istu.edu \\ 3 Melentiev Energy Systems Institute of Siberian Branch, Russian Academy of Science, 664033 Irkutsk, Russia \\ * Correspondence: dsidorov@isem.irk.ru
}

check for

updates

Citation: Sidorov, N.A.; Dreglea, A.I.; Sidorov, D.N. Generalization of the Frobenius Formula in the Theory of Block Operators on Normed Spaces. Mathematics 2021, 9, 3066. https:// doi.org/10.3390/math9233066

Academic Editor: Ioannis K. Argyros

Received: 8 October 2021

Accepted: 26 November 2021

Published: 28 November 2021

Publisher's Note: MDPI stays neutral with regard to jurisdictional claims in published maps and institutional affiliations.

Copyright: (C) 2021 by the authors. Licensee MDPI, Basel, Switzerland. This article is an open access article distributed under the terms and conditions of the Creative Commons Attribution (CC BY) license (https:/ / creativecommons.org/licenses/by/ $4.0 /)$.

\begin{abstract}
The efficient construction and employment of block operators are vital for contemporary computing, playing an essential role in various applications. In this paper, we prove a generalisation of the Frobenius formula in the setting of the theory of block operators on normed spaces. A system of linear equations with the block operator acting in Banach spaces is considered. Existence theorems are proved, and asymptotic approximations of solutions in regular and irregular cases are constructed. In the latter case, the solution is constructed in the form of a Laurent series. The theoretical approach is illustrated with an example, the construction of solutions for a block equation leading to a method of solving some linear integrodifferential system.
\end{abstract}

Keywords: Frobenius formula; operator theory; block operators; Fredholm operator; asymptotic approximations; Nekrasov-Nazarov method

MSC: 47N20; 47A13

\section{Problem Statement}

The Frobenius formula is powerful tool in contemporary applications of linear algebra [1]. The application of the generalized Frobenius formula for solution of singularly perturbed linear equations was considered in [2] readers may refer to lectures [3] for hierarchical matrices ( $\mathcal{H}$-matrices) theory making possible the approximation of certain sub-blocks of a given matrix by low-rank matrices. For the perturbation theory of linear operators, readers may refer to [4-10]. The connection Frobenius formula under the construction of generalized inverse operator matrices (also in the sense of Sherman-Morrison-Woodbury) for the operator matrices was considered in [11,12]. The Frobenius formula is widely in optimization, optimal control problems, and high-performance computing [13-15].

The construction of the approximate methods in bifurcation theory [16] in the case of block operators in Banach spaces also employed the Frobenius formula. The latter studies were motivated by a review of L.A. Lusternik [17] and monograph [18]. The role of skeleton decomposition of linear operators and applications for solution of irregular systems of partial differential equations is discussed in [19]. Nonlinear operator equations with a functional perturbation of the argument of neutral type were studied in [20]. For details on the contemporary theory of differential-operator and kinetic models, including Vlasov-Maxwell, Fredholm, Volterra, Lyapunov-Schmidt branching equations, readers may refer to [9].

Let us now consider real Banach spaces $X_{j}, Y_{i}, j, i=1,2$ and linear bounded operators $A_{i j} \in \mathcal{L}\left(X_{j} \rightarrow Y_{i}\right)$. Consider the following linear equation

$$
A x=y,
$$


where $A=\left[\begin{array}{ll}A_{11} & A_{12} \\ A_{21} & A_{22}\end{array}\right]$ is block operator, $x=\left(x_{1}, x_{2}\right)^{T}, y=\left(y_{1}, y_{2}\right)^{T}, x_{i} \in X_{i}, y_{i} \in Y_{i}$.

Let us introduce the following condition

I. $\quad A_{11}$ is Fredholm operator (see [18], ch. 7, 9), $\operatorname{dim} N\left(A_{11}\right)=n, n \geq 0$.

If $n \geq 1$, then $\left\{\varphi_{i}\right\}_{1}^{n}$ is the basis in $N\left(A_{11}\right),\left\{\gamma_{i}\right\}_{1}^{n}$ is the corresponding biorthogonal system of functionals from $X_{1}^{*},\left\{\psi_{i}\right\}_{1}^{n}$ is the basis in $N\left(A_{11}^{*}\right),\left\{z_{i}\right\}_{1}^{n}$ is the corresponding biorthogonal system of elements from $Y_{1}$.

Let us introduce parameters $\xi_{i}=\left\langle x_{1}, \gamma_{i}\right\rangle$ and outline that for solvability of equation $A_{11} x_{1}+A_{12} x_{2}=y_{1}$ with respect to $x_{1}$ it is necessary and sufficient to fulfil equalities

$$
\left\langle A_{12} x_{2}-y_{1}, \psi_{i}\right\rangle=0, i=1, \ldots, n .
$$

Here $\langle\cdot, \cdot\rangle$ denotes the action of a functional on an element in Banach spaces. In Hilbert spaces, $\langle\cdot, \cdot\rangle$ is replaced by $(\cdot, \cdot)$.

Introduce linear equation

$$
\widehat{A}_{11} x_{1}+A_{12} x_{2}=\sum_{i=1}^{n} \xi_{i} z_{i}+y_{1}
$$

where operator $\widehat{A}_{11}=A_{11}+\sum_{i=1}^{n}\left\langle\cdot, \gamma_{i}\right\rangle z_{i}$ has bounded inverse (see Schmidt's lemma [18], p. 340). Then, from Equation (3) $x_{1}$ can be expressed as a function of $x_{2}$ and parameters $\xi_{1}, \ldots, \xi_{n}$ :

$$
x_{1}=-\widehat{A}_{11}^{-1} A_{12} x_{2}+\sum_{i=1}^{n} \xi_{i} \varphi_{i}+\widehat{A}_{11}^{-1} y_{1} .
$$

Let us substitute $x_{1}$ to the second equation of System (1) and derive the following equation with respect to $x_{2}$

$$
\left(A_{22}-A_{21} \widehat{A}_{11}^{-1} A_{12}\right) x_{2}=y_{2}-A_{21}\left(\widehat{A}_{11}^{-1} y_{1}+\sum_{i=1}^{n} \xi_{i} \varphi_{i}\right) .
$$

Let us introduce the condition:

II. Exists bounded operator

$$
L:=\left(A_{22}-A_{21} \widehat{A}_{11}^{-1} A_{12}\right)^{-1} \in \mathcal{L}\left(Y_{2} \rightarrow X_{2}\right) .
$$

Remark 1. If homogeneous equation $A_{11} x_{1}=0$ has only a trivial solution, and $A_{11}$ is Fredholm operator, bounded inverse operator $A_{11}^{-1}$ then exists, and condition II turns to

$$
\left(A_{22}-A_{21} A_{11}^{-1} A_{12}\right)^{-1} \in \mathcal{L}\left(Y_{2} \rightarrow X_{2}\right) .
$$

This remark holds true for the Frobenius formula, where systems of linear algebraic equations were considered, $X_{1}=Y_{1}=\mathbb{R}^{m}, X_{2}=Y_{2}=\mathbb{R}^{n-m}, \mathbb{R}^{m}, \mathbb{R}^{n-m}$ are arithmetic spaces. Obviously, System (1), as in the case of the classical Frobenius formula for block matrices, can be rewritten as two equations with two unknown vectors, which are elements of different functional Banach spaces.

The aim of this article is to construct solutions of block Equation (1) under Assumptions I, II. The Frobenius formula for block matrices [1] is generalised below.

The remainder of the paper is organized as follows. First, the main theorems dealing with System (1) solvability are considered. Asymptotic approximations of solution are considered next to both in regular and irregular cases. Then, the proposed theory is applied for the solution of an integrodifferential equation. Lastly, concluding remarks are included. The papers uses common definitions and conventional notations from the theory of linear operators; see textbooks [21,22] and review [23]. 


\section{Main Theorems on Solvability of System (1)}

We assumed Conditions I, II as fulfilled, and derived $x_{2}$ from Equation (5)

$$
x_{2}=L\left(y_{2}-A_{21} \widehat{A}_{11}^{-1} y_{1}\right)-\sum_{j=1}^{n} \xi_{j} L A_{21} \varphi_{j} .
$$

In case of $\operatorname{dim} N\left(A_{11}\right)=n$ it remains to determine parameters $\xi_{1}, \ldots, \xi_{n}$. Such parameters can be found from the following system of linear algebraic equations constructed after substitution of $x_{2}$ by virtue of the solvability condition (2), i.e., from system

$$
B \xi=d,
$$

where

$$
B=\left[\left\langle A_{12} L A_{21} \varphi_{j}, \psi_{i}\right\rangle\right]_{i, j=1}^{n}
$$

is $n \times n$ matrix,

$$
\left.d=\left\langle A_{12} L\left(y_{2}-A_{21} \widehat{A}_{11}^{-1} y_{1}\right)-y_{1}, \psi_{i}\right\rangle\right\rangle\left.\right|_{i=1} ^{n}
$$

is vector in $\mathbb{R}^{n}$. Therefore, the following statements is evident.

Theorem 1. Let Conditions I, II be fulfilled. Then, for the unique solvability of system (1) with arbitrary right-hand side, it is necessary and sufficient for matrix B to be nondegenerate.

Corollary 1. Let conditions of Theorem 1 be fulfilled, $\operatorname{det} B \neq 0$; then, operator $A$ is continuously invertible, $\xi=B^{-1} d$ and using Formulae (4) and (6), operator $A^{-1}$ can be explicitly constructed in block form.

Theorem 2. Let conditions I, II be fulfilled, $\operatorname{rank} B=r, B^{*} c_{i}=0, i=1, \ldots, n-r$. If $\sum_{i=1}^{n-r}\left|\left(d, c_{i}\right)\right|=0$, System (1) then has a $n-r$-parametric solution family.

If $\sum_{i=1}^{n-r}\left|\left(d, c_{i}\right)\right| \neq 0$, then system (1) has no solutions.

Proof of Theorems 1 and 2 follows from (4), (6) and known conditions of systems of linear algebraic equations' solvability.

From Theorem 1 it follows generalisation of classic Frobenius formula on the case of block operators in normed spaces.

Corollary 2. Let operators $A_{11}$ and $\left(A_{22}-A_{21} A_{11}^{-1} A_{12}\right)$ be continuously invertible. Then, System (1) has a unique solution, and operator $A^{-1}$ can be constructed explicitly in the block form of Frobenius.

Proof. In that case $x_{2}=\left(A_{22}-A_{21} A_{11}^{-1} A_{12}\right)^{-1}\left(y_{2}-A_{21} A_{11}^{-1} y_{1}\right)$,

$x_{1}=-A_{11}^{-1} A_{12} x_{2}+A_{11}^{-1} y_{1}$. These formulae make it possible to represent the block form of inverse operator $A^{-1}$.

Due to the conditions imposed in Corollary 2, the solution of System (1) is unique and defined as

$$
x=A^{-1} y,
$$

where

$$
\begin{gathered}
A^{-1}=\left[\begin{array}{ll}
M_{11} & M_{12} \\
M_{21} & M_{22}
\end{array}\right], \\
M_{22}=\left(A_{22}-A_{21} A_{11}^{-1} A_{12}\right)^{-1}, \\
M_{21}=-M_{22} A_{21} A_{11}^{-1},
\end{gathered}
$$




$$
\begin{gathered}
M_{11}=A_{11}^{-1}+A_{11}^{-1} A_{12} M_{22} A_{21} A_{11}^{-1}, \\
M_{12}=-A_{11}^{-1} A_{12} M_{22}, \\
M_{i j} \in \mathcal{L}\left(Y_{j} \rightarrow X_{i}\right) .
\end{gathered}
$$

Block operator $A^{-1}$ is a generalisation of Frobenius formula [1] in linear normed spaces.

The following corollary can be similarly verified.

Corollary 3. If $A_{11}$ is Fredholm operator, $\operatorname{dim} N\left(A_{11}\right)=n, \operatorname{det} B \neq 0$, then inverse operator $A^{-1}$ exists and can be explicitly constructed.

Due to the conditions imposed in Corollary 3, the solution of System (1) is unique and defined as

$$
\begin{gathered}
x_{2}=L\left(y_{2}-A_{21} \widehat{A}_{11}^{-1}-A_{21}(\xi, \phi)\right), \\
x_{1}=\widehat{A}_{11}^{-1}\left(y_{1}-A_{21} x_{x}\right)+(\xi, \phi) .
\end{gathered}
$$

Here $(\xi, \phi)=\sum_{1}^{n} \xi_{i} \phi_{i}, \xi=B^{-1} d$. Operators $L$ and $\widehat{A}_{11}^{-1}$, matrix $B$ and vector $d$ (which is linear in $y_{1}$ and $y_{2}$ ) are defined above. The constructed solution, like the result of Corollary 2, can be rewritten in block form $x=A^{-1} y$. This result reinforces the Frobenius approach in the handling of block matrices.

\section{Asymptotic Approximations of Solution}

In this section, the norm of one of operators $A_{21}, A_{12}$ is sufficiently small.

\subsection{Regular Case}

Let the following condition be fulfilled:

III. Assume that each of operators $A_{11}, A_{22}$ have a bounded inverse, $\min \left(\left\|A_{12}\right\|,\left\|A_{21}\right\|\right) \leq \delta$.

Then, without loss of generality, we assume $\left\|A_{12}\right\| \leq \delta$ and consider the following system

$$
A_{11} x_{1}+\lambda A_{12} x_{2}=y_{1}, \quad A_{21} x_{1}+A_{22} x_{2}=y_{2}
$$

with small parameter $\lambda$. For a sufficiently small $\delta>0$, there exists unique solution of such system that can be presented as series expansion in powers of a small parameter $\lambda$.

The substitution of series $x_{1}=\sum_{i=0}^{\infty} a_{i} \lambda^{i}, x_{2}=\sum_{i=0}^{\infty} b_{i} \lambda^{i}$ in system (8), taking into account the invertibility of operators $A_{11}, A_{22}$, coefficients $a_{i}, b_{i}$ can be determined using undefined coefficients method from the following equalities

$$
\begin{aligned}
& \sum_{i=0}^{\infty}\left(a_{i}+\lambda A_{11}^{-1} A_{12} b_{i}\right) \lambda^{i}=A_{11}^{-1} y_{1}, \\
& \sum_{i=0}^{\infty}\left(b_{i}+A_{22}^{-1} A_{21} a_{i}\right) \lambda^{i}=A_{22}^{-1} y_{2} .
\end{aligned}
$$

Therefore, $a_{0}=A_{11}^{-1} y_{1}, b_{0}=A_{22}^{-1} y_{2}-A_{22}^{-1} a_{21} a_{0}, a_{i}=-A_{11}^{-1} A_{12} b_{i-1}, b_{i}=-A_{22}^{-1} a_{21} a_{i}$, $i=1,2, \ldots$ Series convergence in the small neighborhood $|\lambda| \leq \rho$ follows from a wellknown inverse operator theorem (see [21], pp. 141-142). It is easy to estimate the range and speed of convergence.

$$
x_{2}=A_{22}^{-1}\left(y_{2}-A_{21} x_{1}\right),
$$

$x_{1}$ is the solution of equation

$$
\left(A_{11}-\lambda A_{12} A_{22}^{-1} A_{21}\right) x_{1}=y_{1}-\lambda A_{12} A_{22}^{-1} y_{1} .
$$


We fix positive $q<1$ and

$$
\rho=q\left\|A_{11}^{-1} A_{12} A_{22}^{-1} A_{21}\right\|^{-1} .
$$

Then for $|\lambda| \leq \rho$ element $x_{1}$ can be constructed using formula

$$
x_{1}=\sum_{n=0}^{\infty} \lambda^{n}\left(A_{11}^{-1} A_{12} A_{22}^{-1} A_{21}\right)^{n} A_{11}^{-1}\left(y_{1}-\lambda A_{12} A_{22}^{-1} y_{1}\right) .
$$

Because $X_{1}$ is the Banach space, and $q<1$ the series converges for $|\lambda| \leq \rho$ with the rate of a geometrical progression with denominator $q$.

Then the following result is valid.

Theorem 3. Assume that each of operators $A_{11}, A_{22}$ has bounded inverse, and one of operators $A_{21}, A_{12}$ have sufficiently small linear operator norm. Then, operator $A$ has bounded inverse, and System (8) has a unique analytical solution in neighborhood $|\lambda| \leq \rho$.

\subsection{Irregular Case}

Let the following condition be fulfilled:

IV. $A_{11}$ is Fredholm operator, $\operatorname{dim} N\left(A_{11}\right)=1, A_{22}$ invertible operator.

For the sake of clarity, let us make calculations for the case of the system that can be presented as System (8) under condition $\operatorname{dim} N\left(A_{11}\right)=1$.

We prove that, under Condition IV, a solution of System (8) can be represented as the sum of a Laurent series with a pole of order 1 at origin.

Then, we employ the following ansatz

$$
\begin{aligned}
& x_{1} \sim \frac{a_{-1}}{\lambda}+\sum_{i=0}^{\infty} a_{i} \lambda^{i}, \\
& x_{2} \sim \frac{b_{-1}}{\lambda}+\sum_{i=0}^{\infty} b_{i} \lambda^{i} .
\end{aligned}
$$

Coefficients $a_{i}, b_{i}$ can be found using undetermined coefficients method taking into account the Nekrasov-Nazarov method [24].

From equalities

$$
\begin{gathered}
\sum_{i=0}^{\infty}\left(A_{11} a_{i-1}+\lambda A_{12} b_{i-1}\right) \lambda^{i-1}=y_{1} \\
\sum_{i=0}^{\infty}\left(b_{i-1}+A_{22}^{-1} A_{21} a_{i-1}\right) \lambda^{i-1}=A_{22}^{-1} y_{2}
\end{gathered}
$$

the following equations can be derived:

$$
\begin{gathered}
A_{11} a_{-1}=0, \\
b_{-1}=-A_{22}^{-1} A_{21} a_{-1}, \\
A_{11} a_{0}+A_{12} b_{-1}=y_{1}, \\
b_{0}=-A_{22}^{-1} A_{21} a_{0}+A_{22}^{-1} y_{2}, \\
A_{11} a_{i}+A_{12} b_{i-1}=0, \\
b_{i}=-A_{22}^{-1} A_{21} a_{i}, i=1,2, \ldots
\end{gathered}
$$

Due to Fredholm property of operator $A_{11}$, when one solves these equations, it is necessary to take into account the following condition: $\left\langle y_{1}-A_{12} b_{-1}, \psi\right\rangle=0,\left\langle A_{12} b_{i-1}, \psi\right\rangle=0$, $i=1,2, \ldots$ 
Therefore, $a_{-1}=c_{-1} \varphi, b_{-1}=-A_{21}^{-1} A_{21} c_{-1} \varphi$. Constant value $c_{-1}$ can be defined from linear algebraic equality

$$
\left\langle y_{1}+A_{12} A_{22}^{-1} A_{21} c_{-1} \varphi, \psi\right\rangle=0 .
$$

Therefore, we require

$$
\left\langle A_{12} A_{22}^{-1} A_{21} \varphi, \phi\right\rangle \neq 0 .
$$

Then $a_{0}=c_{0} \varphi+\widehat{a}_{0}, b_{0}=A_{21}^{-1} y_{2}-A_{22}^{-1} A_{21}\left(c_{0} \varphi+\widehat{a}_{0}\right)$. Constant value $c_{0}$ can be defined using equality $\left\langle A_{12} b_{0}, \psi\right\rangle=0$. The rest of the coefficients of the Laurent series can be defined in a similar way. The following theorem is true.

Theorem 4. Let $A_{11}$ be Fredholm operator, $\operatorname{dim} N\left(A_{11}\right)=1, A_{11} \varphi=0, A_{11}^{*} \psi=0$, operator $A_{22}$ is invertible. Assume that

$$
\left\langle A_{12} A_{22}^{-1} A_{21} \varphi, \psi\right\rangle \neq 0 .
$$

Then, System (8) in neighborhood $0<|\lambda|<\rho$ has a representable solution as a Laurent series with a first-order pole.

Remark 2. If $\operatorname{dim} N\left(A_{11}\right)=n, n>1,\left\{\varphi_{i}\right\}_{1}^{n}$ is basis in $N\left(A_{11}\right),\left\{\psi_{i}\right\}_{1}^{n}$ is basis in adjoint $N\left(A_{11}^{*}\right)$ and $\operatorname{det}\left\langle A_{12} A_{22}^{-1} A_{21} \phi_{i}, \psi_{j}\right\rangle_{i, j=1}^{n} \neq 0$, then result of Theorem 4 is true.

Proof of Remark 2 repeats the above listed steps of the proof in the case of $n=1$. We seek a solution in the form of a Laurent series with a first-order pole. The coefficients of the series are determined, as before, by the Nekrasov-Nazarov's method. According to the Nekrasov-Nazarov's method, at each step, $n$ arbitrary constants can be determined from $n$ conditions of solvability of the subsequent equations of the recurrent chain of the equations.

Remark 3. If $\operatorname{det}\left\langle A_{12} A_{22}^{-1} A_{21} \phi_{i}, \psi_{j}\right\rangle_{i, j=1}^{n}=0$, then under additional conditions (see [18], sec. 31) solution of system (8) can be constructed as Laurent series with pole of order $p>1$.

Remark 4. The calculation of the coefficients of the Laurent series was carried out for System (1) reduced to Form (8). Obviously, in the case of System (1), reduction to

$$
A_{11} x_{1}+A_{12} x_{2}=y_{1}, \lambda A_{21} x_{1}+A_{22} x_{2}=y_{2} .
$$

under condition of Theorem 4, the coefficients of Laurent series are similarly calculated.

Let us consider the following boundary value problem.

\section{Example 1.}

$$
\left\{\begin{array}{l}
\frac{\partial^{2} u_{1}(x, t)}{\partial x^{2}}+a u_{1}(x, t)+u_{2}(x, t)=y_{1}(x, t) \\
u_{1}(0, t)=0, u_{1}(\pi, t)=0 \\
\lambda \int_{0}^{\pi} K(x, s) u_{1}(s, t) d s+b(x) u_{2}(x, t)=y_{2}(x, t),
\end{array}\right.
$$

here, $y_{1}(x, t), y_{2}(x, t), K(x, s), b(x)$ are known continuous functions for $x \in[0, \pi]$ and $t \in[0, \infty]$, $y_{i}(0, t)=y_{i}(\pi, t)=0, b(x) \neq 0$ for $x \in[0, \pi], \lambda$ is small parmeter.

Let construct the classical continuous solution of BVP (10). Let us introduce operators $\left(A_{11} u_{1}\right)(x, t)=\frac{\partial^{2} u_{1}(x, t)}{\partial x^{2}}+a u_{1}(x, t),\left(A_{12} u_{2}\right)(x, t)=u_{2}(x, t)$, $\left(A_{21} u_{1}\right)(x, t)=\lambda \int_{0}^{\pi} K(x, s) u_{1}(s, t) d s,\left(A_{22} u_{2}\right)(x, t)=b(x) u_{2}(x, t)$. Then, System (10) can be represented in the form of (1).

There are two possible cases: regular $\left(a \neq n^{2}\right)$ and irregular $\left(a=n^{2}\right)$ cases, where $n \in \mathbb{N}^{+}$. 
For the construction of solutions in the regular case, Theorem 3 can be employed, and solutions can be constructed as the following convergent series in the neighbohood of point $\lambda=0$

$$
\begin{aligned}
& u_{1}(x, t)=\sum_{i=0}^{\infty} a_{i}(x, t) \lambda^{i}, \\
& u_{2}(x, t)=\sum_{i=0}^{\infty} b_{i}(x, t) \lambda^{i} .
\end{aligned}
$$

In irregular case $a=n^{2}$, homogeneous boundary problem

$$
\left\{\begin{array}{l}
\frac{\partial^{2} u_{1}}{\partial x^{2}}+n^{2} u_{1}=0 \\
u_{1}(0, t)=u_{1}(\pi, t)=0
\end{array}\right.
$$

has nontrivial solution $c(t) \sin n x$, where $c(t)$ is arbitrary function. Therefore, for solution construction, we employ the Laurent series (see Theorem 4).

The inequality in Theorem 4 is fulfilled if

$$
\int_{0}^{\pi} \int_{0}^{\pi} \frac{K(x, s)}{b(x)} \sin n s \sin n x d x d s \neq 0
$$

Since

$$
u_{2}(x, t)=\frac{1}{b(x)}\left[y_{2}(x, t)-\lambda \int_{0}^{\pi} K(x, s) u_{1}(s, t) d s\right],
$$

for construction of solution it is sufficient to construct the solution of boundary problem

$$
\left\{\begin{array}{l}
\frac{\partial^{2} u_{1}(x, t)}{\partial x^{2}}+n^{2} u_{1}(x, t)-\lambda \int_{0}^{\pi} \frac{K(x, s)}{b(x)} u_{1}(s, t) d s=y_{1}(x, t)-\frac{y_{2}(x, t)}{b(x)} \\
u_{1}(0, t)=u_{1}(\pi, t)=0
\end{array}\right.
$$

as series

$$
u_{1}=\sum_{i=-1}^{\infty} a_{i}(x, t) \lambda^{i}
$$

Coefficients $a_{i}(x, t), i=-1,0,1,2, \ldots$ can be defined as shown in the proof of Theorem 4 using the Nekrasov-Nazarov method [24] two-step method of undetermined coefficients. In this case, the functions of argument $t$ are calculated from the conditions of solvability of the subsequent equations of the recurrent chain of linear equations.

$$
\begin{gathered}
\left\{\begin{array}{l}
\frac{\partial^{2} a_{-1}}{\partial x^{2}}+n^{2} a_{-1}=0, \\
\left.a_{-1}\right|_{x=0}=a_{-1} \mid x=\pi
\end{array}=0,\right. \\
\left\{\begin{array}{l}
\frac{\partial^{2} a_{0}}{\partial x^{2}}+n^{2} a_{0}=\int_{0}^{\pi} \frac{K(x, s)}{b(x)} a_{-1}(s, t) d s+y_{1}(x, t)-\frac{y_{2}(x, t)}{b(x)} \\
\left.a_{0}\right|_{x=0}=\left.a_{0}\right|_{x=\pi}=0 .
\end{array}\right.
\end{gathered}
$$

Then, $a_{-1}(x, t)=c_{-1}(t) \sin n x$, where function $c_{-1}(t)$ can be determined from conditions of solvability of system (14), i.e., from equation

$$
c_{-1}(t) \int_{0}^{\pi} \int_{0}^{\pi} \frac{\sin n x K(x, s)}{b(x)} \sin n s d x d s+\int_{0}^{\pi} \sin n x\left(y_{1}(x, t)-\frac{y_{2}(x, t)}{b(x)}\right) d x=0 .
$$

Solution of boundary problem (14) is following $a_{0}(x, t)=c_{0}(t) \sin n x+\hat{a}_{0}(x, t)$, where $c_{0}(t)$ is arbitrary function, $\hat{a}_{0}(x, t)$ is particular solution of boundary problem (14). Function $c_{0}(t)$ can be similarly defined from the conditions for the solvability of the boundary value problem with respect to the following coefficient $a_{1}(x, t)$ of the Laurent series. 
Remark 5. Results close to Theorem 1 can be found in [11]. The method proposed in [11] requires the prior construction of special projectors and operator constriction. However, the explicit form of the block inverse matrix in the paper is not written out. In our work, the inverse operator is constructed in closed form. A constructive role in our approach is played by the Schmidt-Trenogin pseudoresolvent (operator $\widehat{A}_{11}^{-1}$ ), which has regularizing properties in the Tikhonov-Lavrent'ev sense providing stability of calculations (see [21], sec. 22, pp. 233-237).

\section{Conclusions}

In the general case, when solving block System (1) with a normally solvable operator $A_{11}$, one can use the Laurent series with a higher-order pole. When operator $A_{22}$ is invertible, the problem can be reduced to solution of linear equation $A_{11} x_{1}-B x_{1}=y_{1}-A_{12} A_{22}^{-1} y_{1}$, where $B=A_{12} A_{22}^{-1} A_{21}$. If norm of one of the operators $A_{12}$, $A_{21}$ is sufficiently small, the equation can be written as $A_{11} x_{1}-\lambda B x_{1}=y_{1}-A_{12} A_{22}^{-1} y_{2}$, where $B \in \mathcal{L}\left(Y_{1} \rightarrow X_{1}\right), \lambda$ is small parameter.

If normally solvable operator $A_{11}$ has a complete B-Jordan set and the length of the maximal B-Jordan chain is equal to $p$, then the solution can be constructed in the form of a Laurent series with a pole at a point $\lambda=0$ of order $p$ based on the known facts of perturbation theory. Here readers may refer to book [18], ch. 9, Theorem 31.2.

If the value region of the operator $A_{11}$ is not closed (i.e., the operator is not normally solvable), then we can use regularization methods [10] in the sense of Tikhonov-Lavrent'ev to construct solution of equation.

The approach introduced in this paper can be employed to study complex systems with functional operators $[9,25]$ of various natures.

Author Contributions: Conceptualization, N.A.S. and A.I.D.; Formal analysis, N.A.S., A.I.D. and D.N.S.; Methodology, N.A.S. and A.I.D.; Validation, D.N.S.; Writing—original draft, D.N.S. and N.A.S.; Writing-review and editing, N.A.S. and A.I.D. All authors have read and agreed to the published version of the manuscript.

Funding: This work was funded by the Ministry of Science and Higher Education of the Russian Federation. The research was carried out under State Assignment Project (project no. FWEU-20210006 of the Fundamental Research Program of Russian Federation 2021-2030 using the resources of the High-Temperature Circuit Multi-Access Research Center (Ministry of Science and Higher Education of the Russian Federation, project no 13.CKP.21.0038). This study received no third-party funding.

Institutional Review Board Statement: Not applicable.

Informed Consent Statement: Not applicable.

Data Availability Statement: Not applicable.

Acknowledgments: The authors would like to thank the anonymous reviewers for their constructive suggestions.

Conflicts of Interest: The authors declare no conflict of interest.

\section{References}

1. Gantmacher, F.R. Applications of the Theory of Matrices; Brenner, J.L., Translator; Dover Inc.: New York, NY, USA, 2005.

2. Boglaev, Y.P. The generalized Frobenius formula in singularly perturbed linear equations. Sov. Math. Dokl. 1979, 20, 731-734.

3. Börm, S.; Grasedyck, L.; Hackbusch, W. Hierarchical Matrices; Max-Planck-Institut für Mathematik in den Naturwissenschaften: Leipzig, Germany, 2003.

4. Kato, T. Perturbation Theory for Linear Operators; Springer: Berlin, Germany, 1966.

5. Boichuk, A.A.; Samoilenko, A.M. Generalized Inverse Operators and Fredholm Boundary-Value Problems; Inverse and Ill-Posed Problems Series; Walter de Gruyter GmbH: Berlin, Germany, 2016; Volume 59.

6. Jeribi, A. Spectral Theory and Applications of Linear Operators and Block Operator Matrices; Springer: New York, NY, USA, 2015.

7. Tretter, C. Spectral Theory of Block Operator Matrices and Applications; Imperial Coll. Press: London, UK, 2008.

8. Albeverio, S.; Elander, N. Operator methods in ordinary and partial differential equations. In Proceedings of the Sonja Kovalevsky Symposium, Stockholm, Sweden, 16-22 June 2000; Symposium, S.K., Ed.; Birkhäuser Verlag: Basel, Switzerland, 2002.

9. Sidorov, N.; Loginov, B.; Sinitsyn, A.; Falaleev, M. Lyapunov-Schmidt Methods in Nonlinear Analysis and Applications; Kluwer Academic Publisher: Dordrecht, The Netherlands; Boston, MA, USA; London, UK, 2002; 568p. 
10. Lavrent'ev, M.M.; Savel'ev, L.J. Operator Theory and Ill-Posed Problems; Volume 50 in the Series Inverse and Ill-Posed Problems Series; De Gruyter: Berlin, Germany; Boston, MA, USA, 2011; Volume 50. [CrossRef]

11. Zhuravlev, V.F.; Fomin, N.P.; Zabrodskiy, P.N. Conditions of solvability and representation of the solutions of equations with operator matrices. Ukr. Math. J. 2019, 71, 471-485. [CrossRef]

12. Malozëmov, V.N.; Monaco, M.F.; Petrov, A.V. The Frobenius formula and Sherman-Morrison formulas and related matters. Comput. Math. Math. Phys. 2002, 42, 1403-1409. (Trans. from Russian: Zh. Vychisl. Mat. Mat. Fiz. 2002, 42, 1459-1465).

13. Balandin, D.V.; Kogan, M.M. LMI based output-feedback controllers: $\gamma$-optimal versus linear quadratic. IFAC Proc. Vol. 2008, 41, 9905-9909. [CrossRef]

14. Evtushenko, Y.G.; Zhadan, V.G. Stable barrier-projection and barrier-Newton methods for linear and nonlinear programming. In Algorithms for Continuous Optimization; Springer: Dordrecht, The Netherlands, 1994; pp. 255-285.

15. Erofeev, K.Y.; Khramchenkov, E.M.; Biryal'tsev, E.V. High-performance processing of covariance matrices using GPU computations. Lobacheoskii J. Math.2019, 40, 547-554. [CrossRef]

16. Sidorov, N.A. Explicit and implicit parametrizations in the construction of branching solutions by iterative methods. Sb. Math 1995, 186, 297. [CrossRef]

17. Lusternik, L.A. Some questions of nonlinear functional analysis. Uspehi Mat. Nauk 1956, 72, 145-168.

18. Vainberg, M.M.; Trenogin, V.A. Theory of Branching of Solutions of Nonlinear Equations; Nauka: Moscow, Russia, 1969. (Trans. in English: Wolters-Nordhoff, Leyden, 1974)

19. Sidorov, D.N.; Sidorov, N.A. Solution of Irregular Systems of Partial Differential Equations Using Skeleton Decomposition of Linear Operators; Series "Mathematical Modelling, Programming and Computer Software"; Bulletin of the South Ural State University: Chelyabinsk, Russia, 2017; Volume 10, pp. 63-73. [CrossRef]

20. Sidorov, N.A.; Trufanov, A.V. Nonlinear operator equations with a functional perturbation of the argument of neutral type. Differ. Eqs. 2010, 45, 1840-1844. [CrossRef]

21. Trenogin, V.A. Analyse Fonctionnelle; Mir: Moscow, Russia, 1985. (Trans. from Russian: Funkcional'nyj Analiz; Nauka: Moscow, Russia, 1980)

22. Krantz, S.G. A Guide to Functional Analysis; American Mathematical Soc.: Providence, RI, USA, 2013 ; Volume 49.

23. Gokhberg, I.T.M.G.; Krein, M.G. Fundamental aspects of defect numbers, root numbers and indexes of linear operators. Uspekhi Mat. Nauk 1957, 12, 43-118.

24. Akhmedov, K.T. The analytic method of Nekrasov-Nazarov in non-linear analysis. Uspekhi Mat. Nauk 1957, 12, 135-153.

25. Sidorov, D. Integral Dynamical Models: Singularities, Signals \& Control; World Scientific Series on Nonlinear Science Series A; Chua, L.O., Ed.; World Scientific Publ. Pte: Singapore, 2015; Volume 87. 